\title{
Abordagem Híbrida-Causal Aplicada a Previsão de Deslocamento Horizontal de Bloco de uma Barragem
}

\section{Hybrid-Causal Approach Applied to the Forecast of Horizontal Displacement of a Dam Block}

Jairo Marlon Corrêa1, Tásia Hickmann², Samuel Bellido Rodrigues², Levi Lopes Teixeira²; Étore Funchal de Faria ${ }^{3}$; Edgar Manuel Carreño Franco ${ }^{4}$.

\section{RESUMO}

O presente trabalho propõe o uso de uma metodologia híbrida chamada de ARIMAXGARCH (AXG) cujo objetivo é produzir previsões híbridas para uma série temporal diária de deslocamento de um bloco da Usina Hidrelétrica de Itaipu, localizada em Foz do Iguaçu, Paraná, Brasil. Foi utilizada como variável exógena a série temporal de temperatura no modelo ARIMAX-GARCH, com a finalidade de agregar maior poder de informação em relação ao modelo ARIMA-GARCH. Em todas as análises, a metodologia AXG obteve maior acurácia que a tradicional modelagem ARIMA-GARCH.

Palavras-chave: Segurança de Barragens, Modelos ARIMAX, Modelos GARCH.

\section{ABSTRACT}

The present work proposes the use of a hybrid methodology called ARIMAX-GARCH (AXG) whose objective is to produce hybrid forecasts for a daily time series of displacement of a block of the Itaipu Hydroelectric Power Plant, located in Foz do Iguaçu, Paraná, Brazil. The time series of temperature in the ARIMAX-GARCH model was used as exogenous variable, in order to add more information power in relation to the ARIMA-GARCH model. In all analyzes, the AXG methodology was more accurate than the traditional ARIMA-GARCH modeling.

Keywords: Dam Safety, ARIMAX Models, GARCH Models.
${ }^{1}$ Doutor em Métodos Numéricos em Engenharia e Docente do

Departamento de Matemática e Estatística na Universidade Tecnológica Federal do Paraná UTFPR, Campus Medianeira.

E-mail: jairocorrea@utfpr.edu.br.

${ }^{2}$ Doutor es em Métodos

Numéricos em Engenharia e Docentes do Departamento de Matemática e Estatística na

Universidade Tecnológica Federal do Paraná - UTFPR, Campus Medianeira.

${ }^{3}$ Doutor em Engenharia Elétrica na UNESP-FEIS e Engenheiro da Itaipu Binacional.

${ }^{4}$ Doutor em Engenharia Elétrica e Coordenador do Programa de

Pos-Graduação em Engenharia Elétrica e Computação - PGEEC Unioeste. 


\section{INTRODUÇAOAO}

O uso da energia hidráulica teve ampla importância, desde a origem das civilizações, devido à disponibilidade de recursos, facilidade de aproveitamento e, especialmente, seu caráter renovável. É evidente que a energia hidrelétrica contribuiu efetivamente para o desenvolvimento econômico de forma expressiva como em atividades agrícolas, de serviços, comerciais e, também, no aumento do conforto das residências e avanço da qualidade de vida das pessoas.

A energia hidráulica representa cerca de $20 \%$, atualmente, de toda a eletricidade gerada no planeta; é utilizada como principal fonte na produção de energia elétrica em mais de 30 países, dentre os quais se destacam como maiores produtores mundiais: Canadá, China, Brasil, Estados Unidos e Rússia. Das fontes renováveis é considerada a de maior representatividade na matriz energética mundial por possuir tecnologias aprimoradas, basta considerar as usinas hidrelétricas já construídas (ANEEL, 2008).

A maior usina hidrelétrica do Brasil, em produção de energia elétrica, é a usina binacional de Itaipu, cuja construção foi feita a partir do tratado de Itaipu, no ano de 1973, pelos países vizinhos Brasil e Paraguai. Além disso, a Itaipu Binacional é um dos maiores projetos hidrelétricos do mundo, considerando-se a produção anual. O projeto da barragem da Itaipu foi bastante criterioso tanto em termos de investigação da rocha de fundação quanto dos materiais utilizados na construção das barragens. Além disso foram instalados aproximadamente 2400 instrumentos (ITAIPU, 2009), cujas leituras são feitas desde a época da construção, formando um banco de dados de mais de 30 anos. Em 2005, como parte de modernização da usina, foi implantado um sistema de aquisição automática dos dados, onde $10 \%$ dos instrumentos mais importantes foram automatizados, passando a ter leituras a cada 30 minutos, além da leitura manual já existente, desde o enchimento da barragem, em 1982.

A barragem principal da usina de Itaipu é de concreto, do tipo gravidade aliviada. É composta por 18 blocos, onde estão as tomadas d'água de 16 unidades geradoras (ITAIPU, 2014). A presença de duas forças, a subpressão e pressão na face montante da barragem, faz com que ocorram pequenos deslocamentos horizontais da barragem de concreto, principalmente no sentido montante-jusante. Em contrapartida, as forças estabilizantes ou a favor da segurança são o peso próprio da estrutura e o atrito entre a barragem e a rocha de fundação. De acordo com ITAIPU (2009), foram instalados dois tipos de pêndulos em trechos da Usina Hidrelétrica de Itaipu: o pêndulo direto e o invertido. 
A análise dos dados dos pêndulos mostra que o deslocamento descreve um movimento que gera uma curva cujo comportamento é cíclico, influenciado pela temperatura ambiente. Esses deslocamentos são considerados pequenos e estão dentro dos limites de segurança estabelecidos no projeto. Mesmo assim, a equipe técnica da Itaipu vem acompanhando periodicamente as leituras de todos os instrumentos e incentivado estudos para aplicação de ferramentas matemáticas para atuar preventivamente ante quaisquer riscos potenciais.

Neste trabalho, avalia-se a implementação de um modelo de previsão do deslocamento de um bloco da barragem principal da Usina Hidrelétrica de Itaipu por meio do qual seja possível realizar a análise do comportamento da estrutura em torno deste bloco. Tais informações permitirão medidas corretivas em planos de execução e/ou tomada de decisões já adotadas por engenheiros e técnicos especialistas. Diante disso, também será possível avaliar os demais blocos da usina com a aplicação do método proposto na previsão das demais séries de tempo, uma vez que os resultados poderão operar em benefício da análise do desempenho estrutural da barragem da Usina Hidrelétrica de Itaipu.

Assim sendo, neste artigo, é proposta o uso da metodologia preditiva não-linear híbrida chamada de ARIMAX-GARCH (AXG) cujo objetivo é gerar previsões para uma série temporal de deslocamento de um bloco da Usina Hidrelétrica de Itaipu, localizada em Foz do Iguaçu, Paraná, Brasil. Assim, além da introdução o artigo está dividido em quatro outras seções. Na Seção 2, são abordados os materiais e a metodologia proposta (AXG). Na Seção 3, são apresentados os resultados obtidos via modelo tradicional ARIMA-GARCH contra o modelo proposto AXG. Na Seção 4, é feita a discussão em torno dos resultados obtidos na seção 3, aplicados em uma série temporal de deslocamento de um bloco da barragem da Usina de Itaipu. E, por fim, na Seção 5, o artigo é concluído.

\section{MATERIAIS E METODOS}

Uma série temporal de deslocamento físico de um bloco da barragem que abastece a usina hidrelétrica de Itaipu é modelada devido às suas propriedades estatísticas e à sua relevância. Na figura 1, o seu gráfico mostra o comportamento temporal das 2.554 observações, em intervalos diários. 


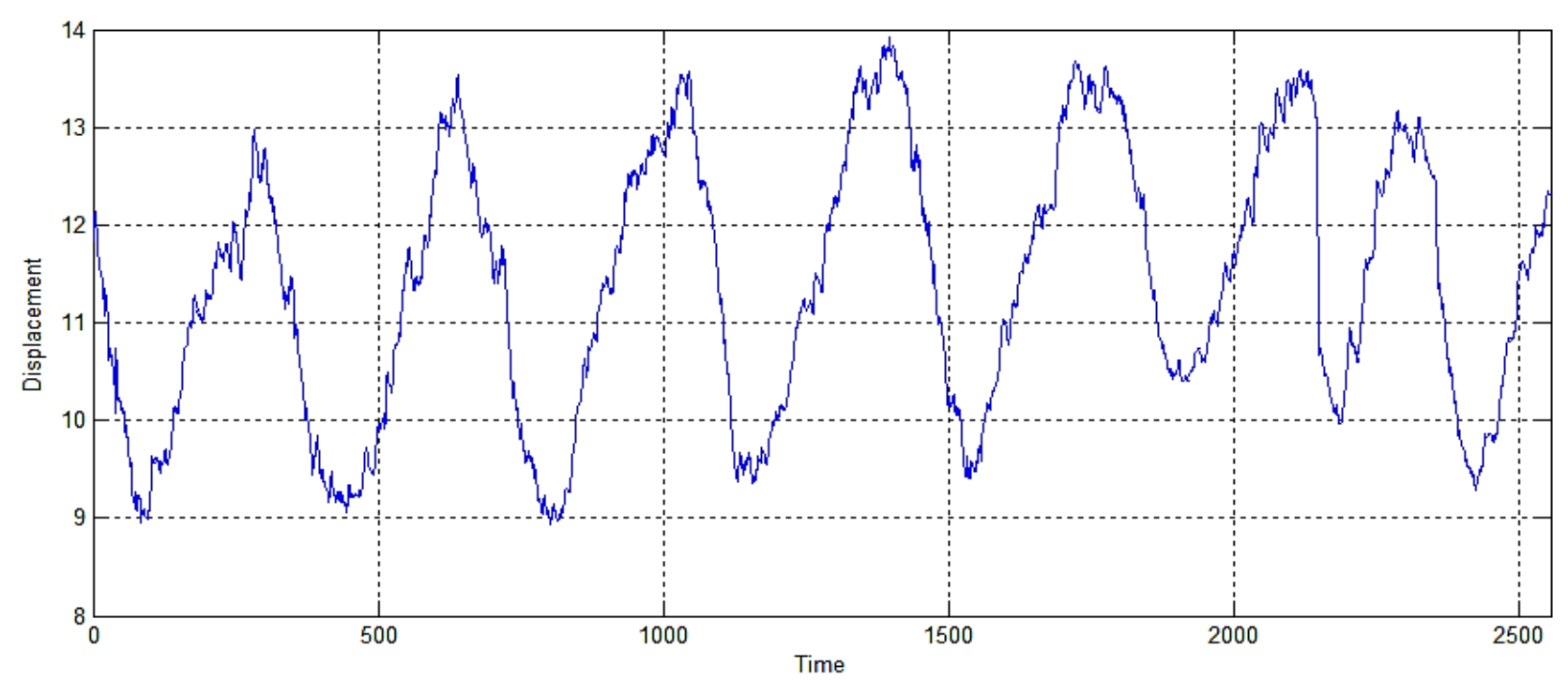

Figura 1 - Série temporal diária de deslocamento físico de um bloco da barragem da usina de Itaipu.

Os referidos dados temporais compreendem o período de 28 de outubro de 2005 a 25 de outubro de 2012, totalizando 2554 pontos, geram uma série temporal. Destes, as 2506 primeiras observações foram utilizadas para obtenção dos parâmetros ótimos dos dois métodos preditivos envolvidos (em outras palavras, tais dados compõem a amostra de treino); enquanto que as 48 observações subsequentes formam a amostra de teste.

Dessa forma, seja $y_{t}(t=1, \ldots, T)$ uma série temporal com estrutura de autodependência linear e não-linear a ser projetada. Baseado em BOX \& TIAO (1975), cada estado $y_{t}$ pode ser representado por um modelo $\operatorname{ARIMAX}(p, d, q)$, conforme em (1).

$$
\nabla^{d} y_{t}=\phi_{0}+\sum_{j=1}^{p} \phi_{j} y_{t-j}+\sum_{j=i}^{q} \theta_{i} e_{t-i}+\sum_{i=1}^{r+1} \sum_{l_{i}=0}^{L_{i}} \alpha_{i l_{i}} x_{i, t-l_{i}}+e_{t}
$$

onde: $B$ é o operador de atraso, definido por $B^{k} y_{t}:=y_{t-k}$, onde $k$ pertence a $\mathbb{Z}$; $\nabla^{d}:=(1-B)^{d}$ é o operador diferença, onde $d$ é a ordem da diferenciação; $\left(\phi_{i}\right)_{i=0}^{p}$ e $\left(\theta_{j}\right)_{j=1}^{q}$ são listas ordenadas de parâmetros complexos, com $\phi_{p} \neq 0$ e $\theta_{q} \neq 0$, e $\alpha_{i l_{i}}$ é o parâmetro complexo associado à componente exógena $x_{i, t-l_{i}}$; $e_{t}$ é uma inovação no tempo t que consiste em uma realização de uma variável aleatória $\varepsilon_{\mathrm{t}}$, onde $\varepsilon_{t}(t=1, \ldots, T)$ é um processo estacionário em média zero e covariância constante; e p e q são, respectivamente, as ordens da parte autoregressiva $\sum_{j=1}^{p} \phi_{j} y_{t-j} \quad$ (notação: $\operatorname{AR}(\mathrm{p})$ ) e da parte de médias móveis $\sum_{j=i}^{q} \theta_{i} e_{t-i}$ (notação: (MA(q)); e $L_{i}$ é a ordem máxima do lag em $\sum_{l_{i}=0}^{L_{i}} \alpha_{i l} x_{i, t-l}$ da variável exógena $x_{i, t}$, onde $i=1, \ldots,(r+1)$. 
Um modelo vastamente utilizado na modelagem da variância condicional é o chamado o Heteroscedasticidade Condicional Autorregressiva (GARCH). Note em (1) que o termo do erro $e_{t}$, em $t$, é a realização de uma variável randômica $\varepsilon_{t}$, com média zero e variância constante, isto é, $\sigma_{t}^{2}=\sigma^{2}$. Em alguns casos a heteroscedasticidade pode depender dos valores observados do passado da série temporal ou de valores passados da sua variância, de tal modo que um modelo $A R$, bem como suas extensões, pode ser adaptado para projetar a variação temporal. Desta forma, incondicionalmente, a variância é constante, mas condicionada a esses valores passados, poderá mudar temporalmente. Segundo BOLLERSLEV (1990), o modelo geral GARCH $(\mathrm{P}, \mathrm{Q})$ para a variância condicional $\sigma_{t}^{2}$ de inovações $\varepsilon_{t}$ é dado em (2).

$$
\sigma_{t}^{2}=\gamma_{0}+\sum_{j=1}^{P} \gamma_{j} \sigma_{t-j}^{2}+\sum_{i=1}^{Q} \delta_{i} e_{t}^{2},
$$

Em (2), as seguintes restrições tem de ser atendidas: $\sum_{j=1}^{P} \gamma_{j}+\sum_{i=1}^{Q} \delta_{i}<1 ; \gamma_{0}>0$; e $\gamma_{j} \geq 0$ $(j=1, \ldots, P)$ e $\delta_{i} \geq 0(i=1, \ldots, Q)$ HAMILTON (1994).

Um modelo composto por (1) e (2) é chamado modelo ARIMAX-GARCH (CORRÊA et al., 2016). Assim, para se obter o melhor modelo ARIMAX-GARCH, três passos básicos devem ser executados: no passo 1 , testar os valores plausíveis para os parâmetros $p, d, q, I, L_{i}$, em (1), e os parâmetros $\mathrm{P}$ e Q em (2), baseado por meio da análise do gráfica das funções de autocorrelação simples e parcial dos erros de previsão padronizados ordinários, $z_{t}(t=1, \ldots, T)$, e quadráticos, $z_{t}^{2}(t=1, \ldots, T)$ (BOLLERSLEV, 1990; ENGLE, 1982); no passo 2, definir o método de estimação dos parâmetros do modelo ARIMAXGARCH (o método mais comum é o de Máxima Verossimilhança (HAMILTON,1994)); e, no passo 3, analisar os testes de diagnósticos para validar (ou não) o modelo ARIMAX-GARCH estimado.

Com o auxílio destas informações, o método AXG consiste, basicamente, na sequência de passos ilustrados no fluxograma da Figura 2. Para a implementação do método AXG, foi utilizado o software MATLAB (versão 2013a) e o software EViews 8. Os dados de temperatura faltantes foram ajustados por meio de interpolação numérica (Spline Cúbica).

No passo 1 do método $A X G$, a série temporal exógena mensal de temperatura ambiente $(t=1, \ldots, 2506)$ foi modelada usando um modelo ARIMA adequado (BOX et al, 2008) , a fim de se produzir as seguintes sequências de previsões, com horizonte de previsão $h=48(t=2507, \ldots, 2554)$. 
No passo 2, a série temporal de temperatura é completada pelas 48 previsões geradas no passo 1. No passo 3, por sua vez, tal variável exógena é utilizada em um modelo ARIMAX-GARCH. Convém salientar que a variável exógena (série de temperatura) foi utilizada com defasagem no tempo. Além disso, a previsão da variância $\hat{\sigma}_{t}^{2}$ forneceu um efeito não-linear para a construção da previsão da média condicional $\hat{y}_{t}$. No passo 4 , são geradas as previsões pelo modelo AXG, para cada instante $t$, chamadas previsões híbridas de $y_{t}(t=1, \ldots, T)$.

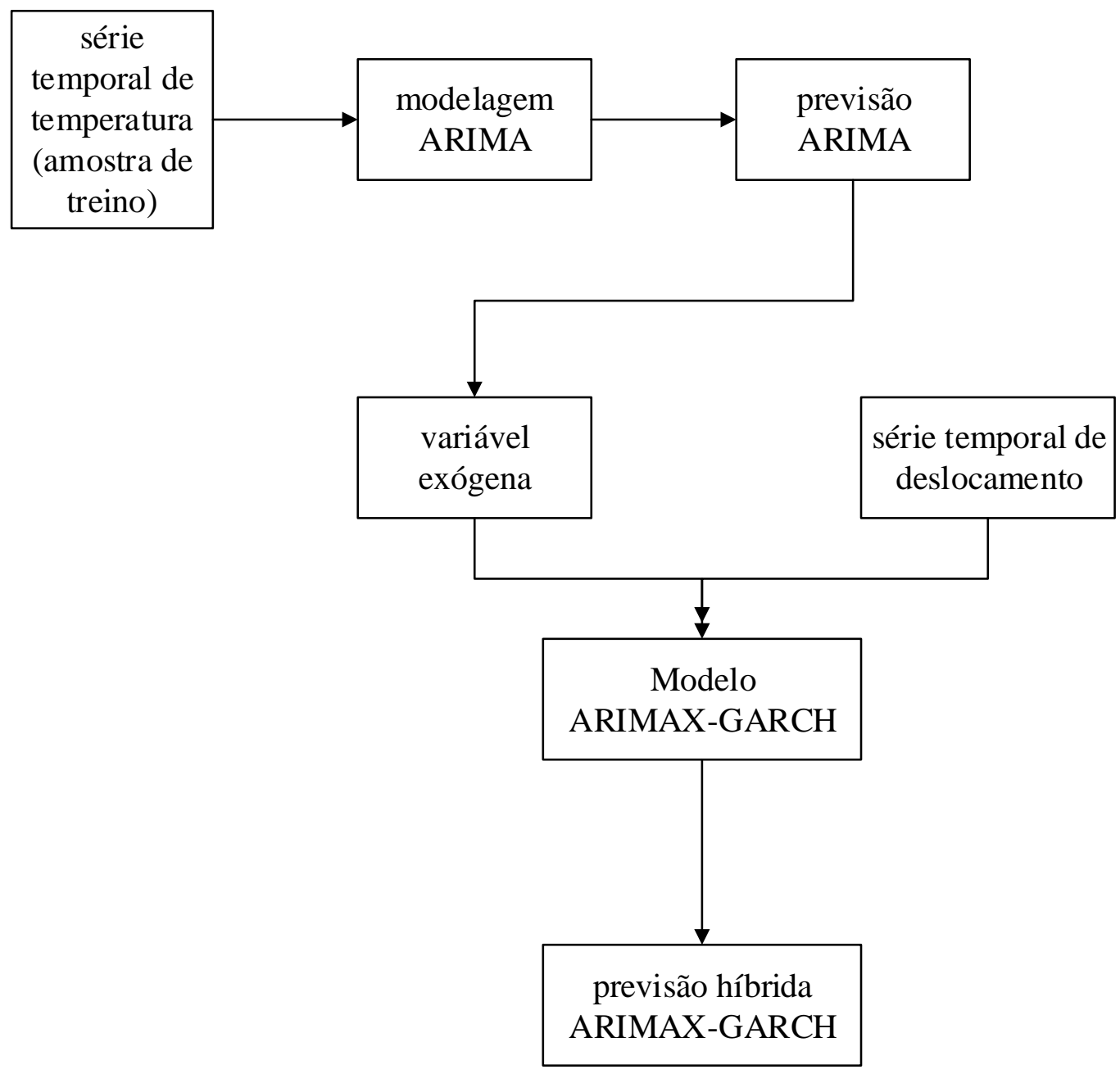

Figura 2 - Fluxograma do método AXG

O método da Máxima Verossimilhança foi usado para se obter as estimativas do modelo ARIMA-GARCH. Todas as estimativas foram estatisticamente significantes, ao nível de 1\%, e satisfaziam às condições de estacionariedade e invertibilidade. Mais detalhes consultar (CORRÊA et al., 2016). 


\section{RESULTADOS E DISCUSSÃO}

Nesta seção, os principais resultados numéricos de uma aplicação do modelo ARIMAGARCH tradicional e do método AXG (proposto) a uma série temporal de aferições diárias do deslocamento de um bloco da barragem da usina de Itaipu são mostrados.

O modelo ARIMA-GARCH (modelo tradicional) com maior aderência à série temporal de deslocamento considerada foi o ARIMA-GARCH $(3,2,4)(2,1)$ (CORRÊA, 2015), com transformação logarítmica seguida por dupla diferenciação. Todas as estimativas de máxima verossimilhança seus parâmetros, tanto da equação da média condicional e quanto da variância condicional, foram estatisticamente significativas, ao nível de $1 \%$ e satisfaziam às condições de estacionariedade e invertibilidade. Em todos os testes residuais, aceitouse a hipótese de inexistência de autocorrelação nos resíduos padronizados ordinários e quadráticos, a 1\% de significância - o que significa que o modelo ARIMA-GARCH capturou uma estrutura de autodependência plausível. Em particular, a estatística Durbin-Watson, calculada a partir dos resíduos ordinários padronizados, obteve valor igual a 2.092367, que sugere a inexistência de autocorrelação de lag 1 , nos resíduos padronizados, na amostra de treino.

Salienta-se ainda que, dentre todos os modelos ARIMA-GARCH plausíveis identificados, foram adotadas, como critério de seleção, as seguintes estatísticas de aderência, na amostra de treinamento: APE (absolute percentage error), MAPE (mean absolute percentage error), MAE (mean absolute error) e $R^{2}$ (explanation coefficient) (HAMILTON,1994)

Para o modelo AXG proposto, foram analisadas as funções FAC e a FACP (até o lag 36) dos resíduos padronizados ordinários e quadráticos. Em todos os lags até 36 , os valores de autocorrelação simples e parcial permaneceram dentro dos intervalos de confiança, com 99\% de credibilidade, o que sugere autocorrelações não diferentes de zero, a 1\% de significância. Baseado na estatística de Ljung-Box sobre os resíduos padronizados ordinários, na amostra de treino, verificou-se que não havia evidência de autodependência linear e quadrática significativas, ao nível de $1 \%$, até a defasagem 36 . O teste $\mathrm{ARCH}$ confirmou inexistência de evidência empírica, ao nível de $1 \%$ de significância, de heteroscedasticidade nos resíduos padronizados ordinários de treino, até o lag 36. Por fim, a estatística Durbin-Watson foi 2.003850 , cujo valor sugere, empiricamente, a inexistência de autocorrelação de lag 1. 
A Tabela 1 fornece os valores obtidos pelas estatísticas de aderência MAPE e MAE, nas amostras de treinamento e de teste.

Tabela 1 - Performance de previsões dos ARIMA-GARCH e AXG.

\begin{tabular}{c|c|c|c|c}
\hline \multirow{2}{*}{ Método } & \multicolumn{2}{|c|}{ MAPE } & \multicolumn{2}{c}{ MAE } \\
\cline { 2 - 5 } & $\begin{array}{c}\text { Dentro da } \\
\text { Amostra }\end{array}$ & Fora da Amostra & Dentro da Amostra & Fora da Amostra \\
\hline ARIMA-GARCH & $0,719 \%$ & $0,506 \%$ & 0,0813 & 0,0602 \\
\hline AXG & $\mathbf{0 , 4 0 1 \%}$ & $\mathbf{0 , 3 0 1 \%}$ & $\mathbf{0 , 0 4 5 0}$ & $\mathbf{0 , 0 3 5 7}$ \\
\hline
\end{tabular}

Note, na Tabela 1, que o método AXG obteve melhor desempenho preditivo em relação ao modelo ARIMA-GARCH, tanto dentro da amostra (amostra de treino) e quanto fora da amostra (amostra de teste). Mais precisamente, tem-se que o método AXG produziu um ganho preditivo relativo sobre o modelo ARIMA-GARCH tradicional, quanto à estatística MAPE, igual a 44,22 \%, na amostra de treino, e igual a 40,51\%, na amostra de teste; enquanto que, em relação à estatística MAE, foi de $44,64 \%$, na amostra de treino, e de $40,69 \%$, na amostra de teste. É desejável que as previsões sejam as mais acuradas possíveis para que os processos decisórios operacionais e financeiros, que por sinal são grandes, sejam executados no momento correto. Salienta-se que a execução de ações operacionais de reparos na barragem ou de investimentos desnecessários ou em períodos inoportunos, pode acarretar, dentre outras coisas, em altas perdas financeiras.

\section{DISCUSSÃO}

Neste artigo, foi proposto novo método preditivo, híbrido e não-linear, para a previsão de séries temporais que utiliza variáveis exógenas de temperatura ambiente. A incorporação de uma variável causal na modelagem da série temporal diária de deslocamento de um bloco da barragem principal da Usina de Itaipu, possibilitou inserir informações na série temporal original, implicando em melhorias substanciais em relação ao modelo de previsão ARIMA-GARCH. Além disso, as previsões agregaram informações oriundas das estruturas lineares (por meio do modelo ARIMA), não-lineares (via modelo GARCH) e da série temporal de temperatura utilizada. Com efeito, o método proposto foi mais informativo e mais acurado que o tradicional modelo ARIMA-GARCH.

A variável exógena (temperatura) bem como suas versões defasadas, tende a apresentar autocorrelação com a série temporal de deslocamento do bloco da barragem, uma vez que a temperatura ambiente influencia no deslocamento estrutural da barragem. 
No presente estudo, a variação de temperatura agregou informações ao modelo $A X G$, melhorando sua capacidade preditiva.

\section{CONSIDERAÇÖES FINAIS}

Houve grande vantagem no quesito poder de predição do modelo proposto AXG contra o modelo tradicional ARIMA-GARCH, conforme os resultados apresentados. $O$ modelo ARIMA-GARCH convencional mostrou que o método $A X G$ não só produziu previsões com melhores performances, de acordo com as estatísticas MAPE e MAE para dados dentro e fora da amostra, conforme apontadas na Tabela 1. Além disso, evidenciase que o método proposto também pode ser operacionalizado de uma forma relativamente simples, com a utilização dos software mencionados no texto.

\section{REFERÉNCIAS}

ANEEL, A. N. D. E. E. Atlas de energia elétrica do Brasil. Agência Nacional de Energia, Brasília, 2008.

BOLLERSLEV, T. Generalized Autoregressive Conditional Heteroscedasticity. Journal of Econometrics, v. 31, p. 307-327, 1990.

BOX, G. E. P.; TIAO, G. C. Intervention Analysis with Applications to Economic and Environmental problems. Journal of American Statistical Association, v. 70, n. 349, p. 70-79, 1975.

CORREA, J. M. Método warimax-garch neural para previsão de séries temporais, 2015. Tese de Doutorado - Universidade Federal do Paraná.

CORRÊA, J. M.; NETO, A. C.; TEIXEIRA JÚNIOR, L. A.; FRANCO, E. M. C.; FARIA, A. E. Time series forecasting with the WARIMAX-GARCH method. Neurocomputing, 2016.

ENGLE, R. F. Autoregressive Conditional Heteroscedasticity with Estimates of the Variance of United Kingdom Inflation. Econometrica, v. 50, n. No. 4, p. 987-1007, 1982.

HAMILTON, J. Time Series Analysis. Princeton University Press, 1994.

ITAIPU. Usina Hidrelétrica de Itaipu: Aspectos de Engenharia. Foz do Iguaçu: TAB Marketing Editurial, 2009.

ITAIPU. Energia hidráulica. Disponível em: <https://www.itaipu.gov.br/energia/energiahidraulica>. Acesso em: 8/5/2014. 